\title{
Analysis of the association between adverse events and outcome in patients receiving a programmed death protein-1 or programmed death ligand-1 antibody
}

\author{
Marilina García-Aranda ${ }^{1,2,3,4}$, Maximino Redondo ${ }^{1,2,3,4}$ \\ ${ }^{1}$ Research Unit, Hospital Costa del Sol, Marbella, Málaga, Spain; ${ }^{2}$ Red de Investigación en Servicios de Salud en Enfermedades Crónicas (REDISSEC), \\ ISCIII, Madrid, Spain; ${ }^{3}$ Instituto de Investigación Biomédica de Málaga (IBIMA), Málaga, Spain; ${ }^{4}$ Surgery, Biochemistry and Immunology \\ Department, School of Medicine, University of Málaga, Málaga, Spain \\ Correspondence to: Maximino Redondo. Research Unit, Hospital Costa del Sol, Marbella, Autovía A-7 km 187, 29603 Málaga, Spain. \\ Email: mredondo@hcs.es. \\ Provenance and Peer Review: This is an invited article commissioned and reviewed by the Academic Editor Dr. Weijun Jiang (Nanjing Normal University, \\ Department of Reproductive and Genetics, Institute of Laboratory Medicine, Jinling Hospital, Nanjing University School of Medicine, Nanjing, China). \\ Comment on: Maher VE, Fernandes LL, Weinstock C, et al. Analysis of the association between adverse events and outcome in patients receiving a \\ programmed death protein 1 or programmed death ligand 1 antibody. J Clin Oncol 2019;37:2730-7.
}

Submitted Nov 08, 2019. Accepted for publication Jan 08, 2020.

doi: $10.21037 /$ cco.2020.03.01

View this article at: http://dx.doi.org/10.21037/cco.2020.03.01

Since their discovery, new immunotherapies have demonstrated their ability to induce durable tumor responses, which have significantly improved long-term survival in patients with different types of metastatic disease. Among the most effective strategies of cancer immunotherapy, the use of monoclonal antibodies against inhibitory immune checkpoint molecules affecting T-cell activation, such as protein death-1 (PD-1) and protein death ligand-1 (PD-L1), represents one of the main investigation areas in cancer research today (1).

Despite the significant benefit that checkpoint inhibitors have represented for metastatic patients, these therapies can promote the activation of autoreactive T-cells resulting in profound inflammatory and unique immune-related adverse events which may be life-threatening and can cause a reduction of the optimal medication dose or treatment discontinuation (2). Although these complications may represent an important impediment for the success of checkpoint blocking therapies, there is a need to understand if the development of autoimmune adverse events may be related with the activation of the immune system and subsequent patient outcome.

In their recent article, Maher et al. evaluate the relationship between the development of adverse events and patient outcome, in terms of response and overall survival, among 1,747 patients with metastatic or locally advanced urothelial cancer who received an anti-PE-1/PD-L1 inhibitor in seven clinical trials that led to product approval by the FDA (3). Provided that systemic corticosteroids could cause immunosuppression and interfere the immune response, they also ask if the use of these substances would affect the duration response.

The authors find that patients who respond to PD-1/ PD-L1 blocking antibodies are more likely to report a related adverse event of special interest (AESI) or related immune-mediated adverse event (imAE) regardless the duration of treatment. They also find that the use of systemic corticosteroids neither appear to affect the chances of developing a response nor duration of response.

As the authors observe, both the duration of observation for AESIs/imAEs and the ability of clinicians involved in cancer patients care to correctly identify which adverse events are actually caused by the use of PD-1/PD-L1 inhibitors represent limiting factors for this type of study which justify the need to develop clinical guides and updated training that allow for the correct definition and management of AESIs/ imAEs related to the corresponding drug.

The authors also highlight the existence of significant differences in the percentage of responding patients with a related AESI/imAE, which leaves an open-ended question 
of whether related AESIs that occur before response and other factors, such as AESI grade, may be predictive of patient outcome, which could have important clinical implications. In this respect, it is necessary to highlight the importance of setting the limits in which adverse events must lead to treatment discontinuation. Accordingly, the development of biomarker panels that allow for patient selection and predict patient response should also be considered, as well as the study of all the different variables that could affect the intensity of related AESIs/imAE, such as drug administration sequence or delay.

Suggested improvements could include the need to improve current understanding of the relationship between systemic immune activation and clinical response to immune therapies in order to develop novel strategies for both patients who are intolerant or refractory to steroids as well as for patients presenting life-threatening adverse events. Basic studies in this area are already showing promising results about the potential value of non-conventional treatments to mitigate AESIs/imAE (4).

Despite the limitations described in the study, provided the large population of patients and the number of trials, their conclusions result convincing and would be useful in clinical practice for patients affected by metastatic or locally advanced urothelial cancer treated with PD-1 or PD-L1 inhibitors.

\section{Acknowledgments}

Funding: None.

\section{Footnote}

Conflicts of Interest: Both authors have completed the ICMJE uniform disclosure form (available at http://dx.doi. org/10.21037/cco.2020.03.01). The authors have no conflicts of interest to declare.

Cite this article as: García-Aranda M, Redondo M. Analysis of the association between adverse events and outcome in patients receiving a programmed death protein-1 or programmed death ligand-1 antibody. Chin Clin Oncol 2020;9(4):57. doi: $10.21037 /$ cco.2020.03.01
Ethical Statement: The authors are accountable for all aspects of the work in ensuring that questions related to the accuracy or integrity of any part of the work are appropriately investigated and resolved.

Open Access Statement: This is an Open Access article distributed in accordance with the Creative Commons Attribution-NonCommercial-NoDerivs 4.0 International License (CC BY-NC-ND 4.0), which permits the noncommercial replication and distribution of the article with the strict proviso that no changes or edits are made and the original work is properly cited (including links to both the formal publication through the relevant DOI and the license). See: https://creativecommons.org/licenses/by-nc$\mathrm{nd} / 4.0 /$.

\section{References}

1. Suarez-Almazor ME, Kim ST, Abdel-Wahab N, et al. Review: immune-related adverse events with use of checkpoint inhibitors for immunotherapy of cancer. Arthritis Rheumatol 2017;69:687-99.

2. Trinh S, Le A, Gowani S, et al. Management of immunerelated adverse events associated with immune checkpoint inhibitor therapy: a minireview of current clinical guidelines. Asia Pac J Oncol Nurs 2019;6:154-60.

3. Maher VE, Fernandes LL, Weinstock C, et al. Analysis of the association between adverse events and outcome in patients receiving a programmed death protein 1 or programmed death ligand 1 antibody. J Clin Oncol 2019;37:2730-7.

4. Perez-Ruiz E, Minute L, Otano I, et al. Prophylactic TNF blockade uncouples efficacy and toxicity in dual CTLA-4 and PD-1 immunotherapy. Nature 2019;569:428-32. 